\title{
UN HERBARIO DE COSTA RICA LLEGA A 75 AÑOS
}

\author{
Carlos O. Morales
}

\author{
Escuela de Biología, Universidad de Costa Rica, 2060 San José, Costa Rica
}

\begin{abstract}
The name of one of its founders, Dr. Luis A. Fournier, was given to the Herbarium of the University of Costa Rica (USJ) in December 2005. During 75 years (1931-2006) this herbarium has contributed to the botanical research in Costa Rica. An update of the vascular plant type catalogue of USJ is included here.
\end{abstract}

Resumen. Desde diciembre de 2005, el Herbario de la Universidad de Costa Rica (USJ) lleva el nombre de uno de sus fundadores: el Dr. Luis A. Fournier. En 2006 este herbario cumple 75 años de contribuir con la investigación botánica en Costa Rica. Se incluye una actualización del catálogo de tipos de plantas vasculares de USJ.

Palabras clave / Key words: Herbario Luis A. Fournier (USJ), Universidad de Costa Rica, especímenes tipo, Type specimens

En diciembre de 2005, en un acto oficial llevado a cabo en la Escuela de Biología de la Universidad de Costa Rica, se asignó el nombre del Dr. Luis A. Fournier Origgi al Herbario de la Universidad de Costa Rica (USJ). Datos biográficos sobre Fournier se hallan en Morales (2002) y en otras fuentes. Sobre el trabajo de Fournier como agrónomo y como biólogo, el Dr. Jorge León ofreció una conferencia en el acto oficial mencionado (el texto de esa conferencia se incluye en este número de Lankesteriana, p. 29-32).

El Herbario Luis A. Fournier (USJ) fue fundado en 1931, antes de que existiera la Universidad de Costa Rica (U.C.R.). Así consta en el Informe Anual de 1931 del Centro Nacional de Agricultura (C.N.A.), que estaba ubicado en terrenos que a partir de 1940 ocupó la U.C.R., en Montes de Oca, San José. En ese informe, el jefe de la Sección de Botánica, José María Orozco, escribió: "Con las plantas que se encuentran en el Campo de Experimentación se ha comenzado a formar la base para un herbario ... Sería de gran utilidad la obtención de un aparato sencillo con el que se puedan envenenar periódicamente los ejemplares coleccionados sin tener que recurrir al tratamiento de ellos uno por uno, pues no ha bastado someterlos a la acción del sol; en corto tiempo hemos tenido que retirar un gran número de ejemplares destruidos por hongos e insectos". Muchos de los especímenes preparados por Orozco desaparecieron, no solamente por la acción de hongos e insectos, sino también a causa de un incendio que ocurrió 34 años después, el 20 de marzo de 1965. Hasta 1965 hubo una secadora de plantas junto a las colecciones, en un edificio de madera sin ninguna protección contra incendios; el contacto directo entre las bombillas de la secadora y unos especímenes secos, más el calor de marzo, provocaron la pérdida de miles de especímenes y de la biblioteca del herbario (L.A. Fournier, com. pers. ca. 2000). En la actualidad, USJ cumple con normas internacionales de seguridad en colecciones científicas; así, por ej., el error de tener una secadora dentro del herbario no se comete nunca más.

Este herbario posee colecciones muy importantes de los principales forjadores a lo largo de siete décadas y media: José María Orozco, Rafael Lucas Rodríguez, Luis A. Fournier, Sergio Salas, Luis Poveda, Dora Emilia Mora y Jorge Gómez Laurito. USJ posee, además, colecciones muy valiosas de Robert L. Dressler, Mario A. Blanco, Franco Pupulin, José A. Sáenz, Julieta Carranza, Peter Döbbeler y Jorge León. Más información sobre USJ, sus colecciones y sus curadores actuales se halla en la página de la Escuela de Biología en Internet: http://biologia.ucr.ac.cr/ herbario.html.

La base de datos de USJ, que todavía no se ha concluido, estará disponible en Internet en un plazo de unos tres años, en el marco de un convenio firmado en 2005 entre el Instituto Nacional de Biodiversidad (INBio) y la Escuela de Biología. En este esfuerzo regional para compartir información científica también participan otros herbarios de América Central, con financiamiento de la Agencia Noruega de Cooperación para el Desarrollo (NORAD).

En mayo de 2006 se concluyó la instalación de un sistema de compactadores, que consta de seis archivos movibles manualmente, montados sobre cuatro rieles (Fig. 1). Toda la colección de dicotiledóneas, que es la más grande del herbario, se halla en este sistema. Con esta novedosa estructura, las colecciones de plantas 
vasculares prácticamente han duplicado la capacidad de uso del espacio disponible.

Además de tener una representación significativa de la flora de Costa Rica, USJ alberga numerosos especímenes de diversos países, sobre todo de América y Europa. El intercambio de especímenes con herbarios de otros países ha sido muy fructífero en la historia de USJ. Los primeros países representados fueron EE.UU., México y Argentina; posteriormente se recibió material de los países centroamericanos (excepto Belice y Nicaragua) y pocos especímenes de Sudamérica y de las Antillas (sobre todo Jamaica y Puerto Rico). En los últimos años se ha recibido material de Cuba, Alemania, Argentina de nuevo, Austria e Italia, entre otros países menos representados. En total, USJ posee especímenes de $c a .30$ naciones de seis grandes regiones del planeta. En los últimos cinco años, el intercambio ha sido especialmente fructífero con $\mathrm{M}$ (especímenes de USJ a cambio de literatura), ULM y BREM (los tres de Alemania), CTES (Argentina) y CM (EE. UU.). Desde Corrientes, en 2005 se recibieron especímenes de Argentina, Paraguay, Brasil y Bolivia. En enero de 2006 se recibieron duplicados de Carnegie Museum, entre ellos algunos ya montados de C.G. Pringle y otros recolectores, que datan de las últimas décadas del siglo XIX; el más antiguo es un espécimen de Cyperus esculentus L. (Cyperaceae), recolectado por B.H. Patterson en Pennsylvania el 16 de septiembre de 1876. Este es el espécimen más antiguo que posee USJ. La mayoría son plantas norteamericanas recolectadas desde México hasta el norte de Canadá, con algunas especies de Panamá y Belice (British Honduras; éstas recolectadas por Percy H. Gentle en 1937 y 1938). En USJ mantenemos el interés por todos los grupos de plantas, algas, hongos y líquenes y desearíamos tener especímenes de todas las regiones del mundo. Hasta ahora, África, Asia y Polinesia no están representadas en este herbario.

Actualmente, USJ tiene representados todos los grupos que tradicionalmente ha estudiado la botánica: espermatófitos, pteridófitos, briófitos, algas, hongos y líquenes; en total son $c a .90000$ ejemplares registrados. Cerca de dos tercios de las colecciones son plantas vasculares de Costa Rica. Un catálogo de los tipos de plantas vasculares de USJ, que incluye un resumen de la historia de este herbario, fue preparado por Morales \& Villalobos (2004). Desde la aparición de este catálogo, USJ ha recibido 21 tipos más y siete ilustraciones de tipos. [También se recibió un clonotipo orquidáceo ex AMO: Epidendrum synchronum Hágsater, Icon.
Orchid. 7: Lám. 793. 2004. Costa Rica: San José, Cordillera Central, La Palma, al norte de San Jerónimo de Moravia, 1450 m, 20 Dec 1982, clonotype pressed 15 Jun 1984, E. Hágsater 6990 (CT: USJ), con una separata de la publicación original]. Así, el número total de tipos (hasta agosto de 2006) es de 244; de éstos, 77 son holotipos, 104 isotipos, 61 paratipos y 2 neotipos; estos últimos fueron indicados erróneamente como lectotipos en el catálogo referido: Myoxanthus vittatus Pupulin \& M.A. Blanco y Trichocentrum costaricense Mora-Ret. \& Pupulin. Recientemente, una revisión cuidadosa de los tipos de Orchidaceae y de todo el material en proceso confirmó que USJ no recibió los holotipos de estas dos especies citados en la literatura; por tanto, las neotipificaciones de Morales \& Villalobos (2004) permanecen válidas. Además, el herbario cuenta con 113 ilustraciones de tipos (fotos, fotocopias y dibujos). En suma, 51 familias de plantas vasculares de este herbario poseen tipos o, al menos, ilustraciones de tipos. Con 114 tipos, la familia Orchidaceae abarca el $47 \%$ de los tipos de USJ. Es justo mencionar que 11 de los 21 tipos registrados en el último año y medio son de Orchidaceae, especialmente por el intenso trabajo taxonómico que realiza Franco Pupulin en el Jardín Botánico Lankester.

A continuación se detallan los tipos recibidos y anotados desde enero de 2005 hasta agosto de 2006, para actualizar el catálogo de Morales \& Villalobos (2004):

\section{PTERIDOPHYTA}

\section{POLYPODIACEAE}

Campyloneurum gracile A. Rojas, Lankesteriana 5(1): 41. 2005. Costa Rica: San José; Tarrazú, San Carlos, Reserva Biológica Ríos Paraíso, fila al oeste de Río Negro, 9³3'30”N, 8408'10”W, 500 m, 18 mayo 2004, A. Rojas \& M. Hidalgo 5666 (HT: CR, IT: K, MO). Un isotipo en USJ.

\section{SPERMATOPHYTA}

\section{Asclepiadaceae}

Gonolobus ustulatus W.D. Stevens, Novon 15(1): 242. 2005. Costa Rica: Alajuela; Reserva Forestal de San Ramón, $10^{\circ} 13^{\prime} \mathrm{N}, 84^{\circ} 37^{\prime} \mathrm{W}, 900-1200$ m, 12-15 mar 1987, J. Gómez Laurito 11413 (HT: F). Un isotipo en USJ.

Bombacaceae

Quararibea gomeziana W.S. Alverson, Brittonia 
41(1): 61. 1989. Costa Rica: Limón; Río Sand Box, ca. $0.5 \mathrm{~km}$ upstream from Bribri-Cahuita rd. crossing, 9³8'47'N, 82 49'28'W, 90 m, 28 Oct 1983, W.S. Alverson 2136 (HT: WIS, IT: B, BR, COL, CR, DUKE, F, G, GH, HUA, K, MO, NY, PMA, S, UC, US, USJ, Z). Un segundo isotipo ex WIS se halla en la colección de USJ (incluye fotocopia de una flor).

\section{Campanulaceae}

En este caso, se hace una corrección:

Burmeistera quercifolia Gómez-Laur. \& L.D. Gómez, Brenesia 63-64: 127. 2005. Costa Rica: Alajuela; San Carlos, Quesada, Cerros Volcán Viejo, $10^{\circ} 14^{\prime} 12^{\prime \prime} \mathrm{N}, 83^{\circ} 18^{\prime} 27^{\prime \prime} \mathrm{W}, 2200 \mathrm{~m}, 11$ nov 1983 (fl, fr), J. Gómez Laurito 9677 (HT: USJ, IT: CR, F).

Morales \& Villalobos (2004) citan este espécimen como paratipo, tal como se indicaba en la etiqueta de herbario. Sin embargo, en la publicación original (Brenesia 22: 352. 1984) no se mencionó el holotipo, tampoco recolector ni número. Por eso, el nombre de la especie fue un nom. nudum hasta 2005.

\section{Dichapetalaceae}

Dichapetalum reliquum Kriebel \& Al. Rodr., Lankesteriana 5(2): 134. 2005. Costa Rica: Puntarenas; Coto Brus, San Vito, Wilson Botanic Garden, Las Cruces Biological Station, $8^{\circ} 47^{\prime} 08^{\prime \prime} \mathrm{N}$, 8257'29”'W, 1290 m, 27 Jun 1994, W.J. Kress 944552 (PT: CR). También un paratipo en USJ.

\section{LENTIBULARIACEAE}

Utricularia uxoris Gómez-Laur., Lankesteriana 5(2): 137. 2005. Costa Rica: Cartago; Jiménez, Pejibaye, Selva, Reserva Biológica El Copal,

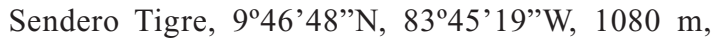
23 oct 2004 (fl, fr), J. Gómez Laurito et al. 14360 (HT: USJ, IT: CR, F). Loco typico, 5 sept 2003 (fl), J. Gómez Laurito et al. 14088 (PT: USJ); 18 jun 2005 (botones flor.), J. Gómez Laurito et al. 14469 (PT: USJ).

\section{Malvaceae}

Monteiroa reitzii Krapov., Bonplandia (Corrientes) 12: 58-60. 2003. Brasil: Santa Catarina; 9 km E de Bom Jardim da Serra, 25 nov 1980, A. Krapovickas \& R. Vanni $36928 A$ (PT: USJ). Un paratipo ex CTES.

\section{Myrtaceae}

Eugenia herrerae Barrie, Novon 15(1): 24-26. 2005. Costa Rica: Limón; Guápiles, Los Ángeles, 24 febr 1990 (fl), G. Herrera \& S. Schik 3790 (HT:
CR, IT: F, K, MO). Un isotipo en USJ.

\section{ORCHIDACEAE}

Brassia suavissima Pupulin \& Bogarín, Orchids (West Palm Beach) 74(3): 206. 2005. Costa Rica: San José; Pérez Zeledón, Páramo, San Ramón Sur, toward Río Berlín, ca. $9^{\circ} 25^{\prime} \mathrm{N}, 8^{\circ} 44^{\prime} \mathrm{W}, 1420-1640 \mathrm{~m}$, collected by Esteban Víquez J., flowered in cultivation in the collection of Marta Herra, 29 May 2004, F. Pupulin 5236 (HT: USJ).

Crossoglossa latifolia Pupulin, ined., Costa Rica: Alajuela; San Ramón, Ángeles, Reserva Biológica Alberto M. Brenes, orillas del Río San Lorencito, cerca del Sendero Terciopelo, $10^{\circ} 13^{\prime} 06^{\prime \prime} \mathrm{N}, 84^{\circ} 36^{\prime} 11^{\prime \prime} \mathrm{W}$, 800-900 m, 22 mayo 2004, floreció en cultivo en el Jardín Botánico Lankester, 15 mayo 2005, D. Bogarín 817 (HT: USJ).

Dichaea viridula Pupulin, Harvard Pap. Bot. 10(1): 85. 2005. Costa Rica: Cartago; Paraíso, Orosi, Tapantí National Park, Oropéndola trail, 1160 m, 25 May 2003, F. Pupulin 4752 (PT: USJ).

Encyclia ossenbachiana Pupulin, ined., Costa Rica: Cartago; Tierra Blanca, Río Reventado, along the river edge, collected by Rafael A. González, Jul 2001, flowered in cultivation at Jardín Botánico Lankester, 8 Jul 2003, D. Bogarín 357 (HT: USJ). Holotipo más lámina con foto in vivo y dibujos del holotipo.

Epidendrum atwoodii Hágsater \& L. Sánchez, Icon. Orchid. (México) 3: Lám. 311. 1999. Costa Rica: Cartago; Turrialba, sin localidad precisa, comprada y cultivada por V. Gin Lun, V. Gin Lun s.n. (HT: USJ38234). Es un ejemplar seco montado, con una flor en líquido; incluye separata con ilustración original.

Epidendrum x monteverdense (Pupulin \& Hágsater) Hágsater, Lankesteriana 5(1): 74. 2005. Costa Rica: San José; fila de la Cordillera de Talamanca [Desamparados, San Cristóbal], 1 km al sur de Casa Mata, 1950 m, preparado de material cultivado, 18 febr 1985, E. Hágsater \& C. Horich 6320 (USJ: PT). Paratipo y separata con ilustraciones ex AMO. Basiónimo: Oerstedella x monteverdensis Pupulin \& Hágsater. El holotipo en USJ ya fue citado en el catálogo.

Lepanthes gerardensis M.A. Blanco, Lankesteriana 8: 19-21. 2003. Una lámina con ilustraciones originales del holotipo (que ya fue citado en el catálogo).

Prosthechea barbozae Pupulin, Selbyana 25(1): 20. 2004. Lámina con fotografías de planta, flores y partes florales. El holotipo en USJ ya fue citado en el catálogo.

Sigmatostalix dulcineae Pupulin \& G. Rojas, ined., Costa Rica: Puntarenas; Coto Brus, Las Alturas de Cotón, Fila Cedro, 859'03”N, 82 $54^{\circ} 20^{\prime \prime} \mathrm{W}, 1240$ - 
$1670 \mathrm{~m}, 20$ Mar 2003, flowered in cultivation at Jardín Botánico Lankester, 17 May 2004, F. Pupulin et al. 4405 (PT: USJ).

Sigmatostalix dulcineae forma flava Pupulin \& G. Rojas, ined., Costa Rica: Puntarenas; Coto Brus, Las Alturas de Cotón, Fila Cedro, 859'03”N, 8254'20'”, $1240-1670$ m, 20 Mar 2003, flowered in cultivation at Jardín Botánico Lankester, 23 May 2005, F. Pupulin et al. 4406 (HT: USJ).

Sigmatostalix pseudounguiculata Pupulin \& Dressler, Lindleyana 15(1): 27. 2000. Un isotipo en USJ (además del holotipo, que ya fue citado en el catálogo).

Stanhopea confusa G. Gerlach \& Beeche, Lankesteriana 4(3): 217. 2004. Costa Rica: Cartago; Turrialba, vertiente este de la Cordillera de Talamanca, entre Grano de Oro y Moravia de Chirripó, 800-900 m, 13 jul 1993, floreció en el Jardín Botánico de MúnichNymphenburg (No. 93/3084), sept 2000, C.K. Horich s.n. (HT: USJ-86514). Costa Rica: Heredia; Cariblanco, camino a Colonia Virgen del Socorro, 850 m, 1 jul 1995, M.A. Blanco \& A. Vega 345 (USJ: PT). Gerlach (loc. cit.: 220) citó este espécimen erróneamente como "Blanco 51".

Trichocentrum obcordilabium Pupulin, Novon 8(3): 238. 1998. Una lámina con ilustraciones originales del tipo. Un isotipo en USJ ya fue citado en el catálogo.

\section{LITERATURA CITADA}

Morales, C.O. 2002. Ecce homo, scientia clarus: Luis Fournier Origgi (1935-2002). Lankesteriana 5: 1-2. Morales, C.O. \& Natalie Villalobos T. 2004. Tipos de plantas vasculares en el Herbario de la Universidad de Costa Rica (USJ). Lankesteriana 4(3): 187-208.

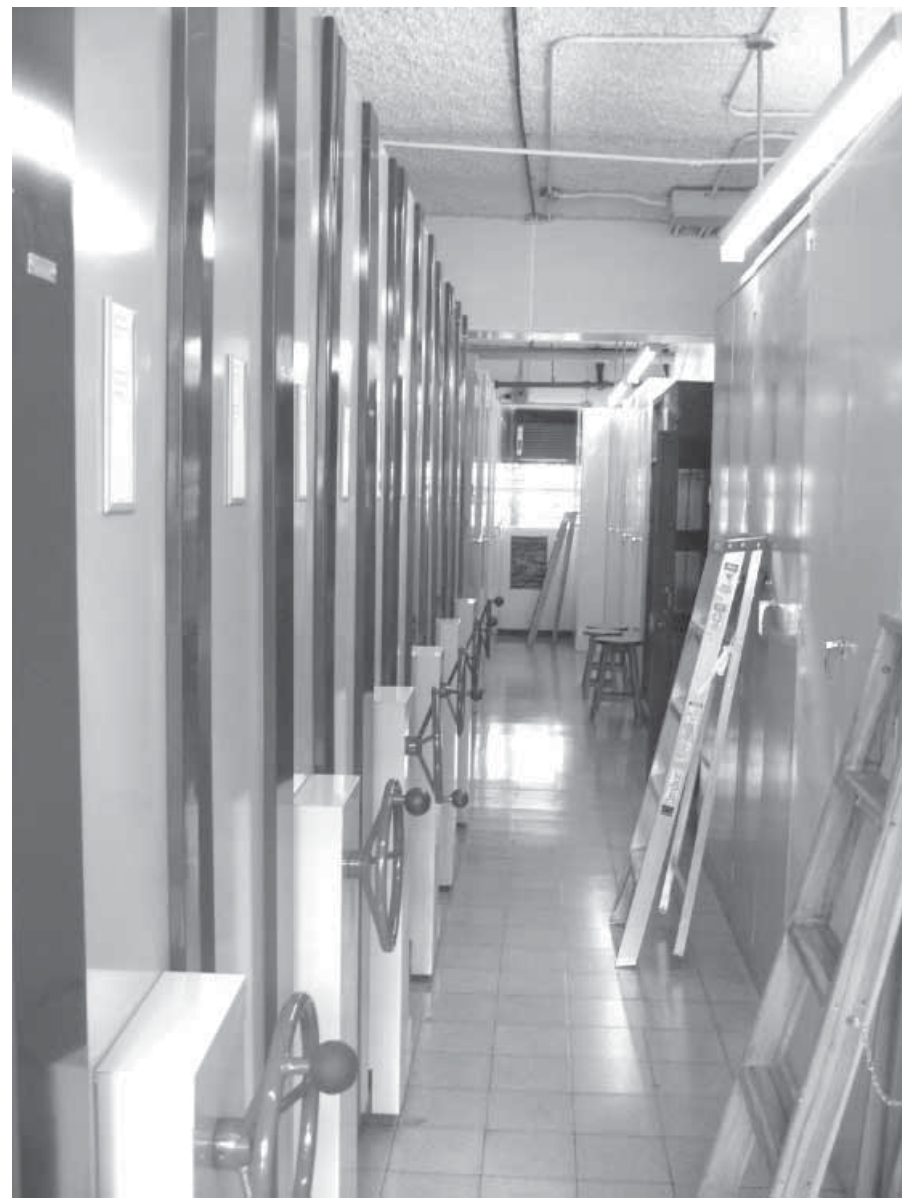

Fig. 1. Vista general del sistema de compactadores (izquierda), que alberga desde mayo de 2006 la colección de dicotiledóneas de USJ. Foto: Elmer G. García, junio de 2006. 